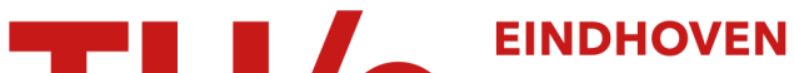 \\ UNIVERSITY OF \\ TECHNOLOGY
}

\section{Control design for a mobile robot Including tire behavior}

Citation for published version (APA):

Ploeg, J., Schouten, H. E., \& Nijmeijer, H. (2008). Control design for a mobile robot Including tire behavior. In Proceedings of the IEEE Intelligent Vehicles Symposium (IV'2008), 4-6 June 2008, Eindhoven, The Netherlands (pp. 240-245). Institute of Electrical and Electronics Engineers. https://doi.org/10.1109/IVS.2008.4621292

DOI:

10.1109/IVS.2008.4621292

Document status and date:

Published: 01/01/2008

\section{Document Version:}

Accepted manuscript including changes made at the peer-review stage

\section{Please check the document version of this publication:}

- A submitted manuscript is the version of the article upon submission and before peer-review. There can be important differences between the submitted version and the official published version of record. People interested in the research are advised to contact the author for the final version of the publication, or visit the $\mathrm{DOI}$ to the publisher's website.

- The final author version and the galley proof are versions of the publication after peer review.

- The final published version features the final layout of the paper including the volume, issue and page numbers.

Link to publication

\section{General rights}

Copyright and moral rights for the publications made accessible in the public portal are retained by the authors and/or other copyright owners and it is a condition of accessing publications that users recognise and abide by the legal requirements associated with these rights.

- Users may download and print one copy of any publication from the public portal for the purpose of private study or research.

- You may not further distribute the material or use it for any profit-making activity or commercial gain

- You may freely distribute the URL identifying the publication in the public portal.

If the publication is distributed under the terms of Article $25 \mathrm{fa}$ of the Dutch Copyright Act, indicated by the "Taverne" license above, please follow below link for the End User Agreement:

www.tue.nl/taverne

Take down policy

If you believe that this document breaches copyright please contact us at:

openaccess@tue.nl

providing details and we will investigate your claim. 


\title{
Control Design for a Mobile Robot Including Tire Behavior
}

\author{
Jeroen Ploeg, Hanno E. Schouten, Henk Nijmeijer
}

\begin{abstract}
In order to support the development process of Advanced Driver Assistance systems for road vehicles, TNO is operating a hardware-in-the-loop test setup. In this facility, called VeHIL, vehicles in the direct neighborhood of the test vehicle are simulated using wheeled mobile robots. Due to the required type of maneuvers, these robots have independently driven and steered wheels. Consequently, the robot is overactuated. Furthermore, since the robot is capable of high dynamic maneuvers, slip effects caused by the tires can play an important role. A position controller based on feedback linearization is presented, using the so-called multicycle approach which regards the robot as a set of independent unicycles. As a result, the wheeled mobile robot is position controlled while each unicycle is controlled taking weight transfer as well as longitudinal and lateral tire slip into account.
\end{abstract}

Index Terms-Mobile robots, Nonlinear control, Robotics

\section{INTRODUCTION}

Advanced Driver Assistance systems such as Adaptive Cruise Control, Collision Warning and Mitigation, are increasingly available in road vehicles. In order to support the development of these systems, TNO Automotive has built its VeHIL test facility [1]. VeHIL (Vehicle Hardware-In-theLoop) enables the hardware-in-the-loop testing of entire road vehicles equipped with driver assistance functionality based on environment sensors such as radar, lidar or vision. The principle of VeHIL is to simulate the relative motion of other vehicles with respect to the test vehicle, allowing for efficient, safe and reproducible testing. The neighboring vehicles are simulated by wheeled mobile robots (WMR's), one of which is shown in Fig. 1. This VeHIL WMR or Moving Base (MB) differs from most wheeled robots used in industry, in that it is a high dynamic robot, capable of extreme maneuvers at velocities up to $50 \mathrm{~km} / \mathrm{h}$ in all directions. Consequently, the control system has to be designed such that these high dynamic specifications are fully exploited, which is the focus of this paper.

The main control objective of the MB is to let its center track a reference trajectory consisting of the position in the horizontal plane and the orientation. This is achieved by four independently driven and steered wheels. As a consequence, the MB has eight actuators - four driving and four steering motors - whereas the control objective comprises three degrees of freedom only. The MB can therefore be characterized as being overactuated. In [2] a

J. Ploeg and H. E. Schouten are with TNO Science and Industry, Business Unit Automotive, P.O. Box 756, 5700 AT Helmond, The Netherlands, \{jeroen.ploeg, hanno.schouten\}@tno.nl

$\mathrm{H}$. Nijmeijer is with the Eindhoven University of Technology, Mechanical Engineering Department, Eindhoven, The Netherlands, h.nijmeijer@tue.nl

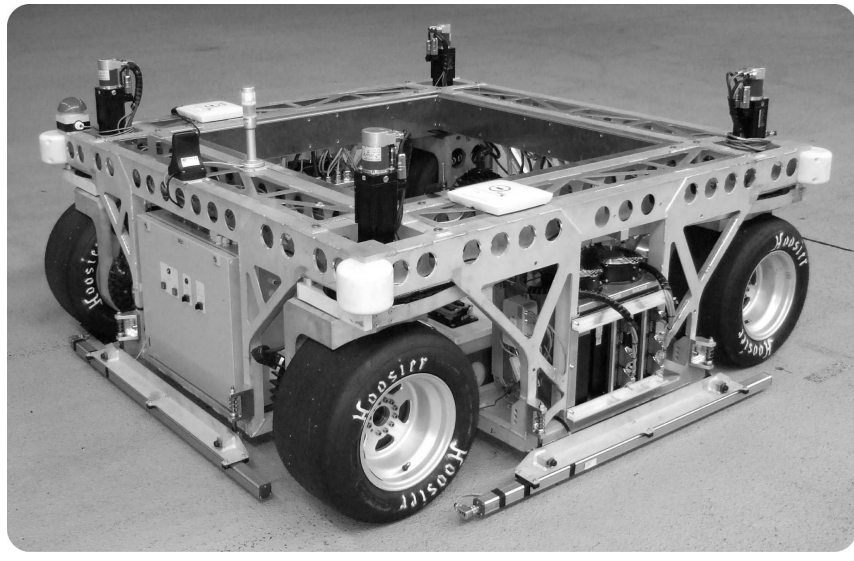

Fig. 1. TNO's wheeled mobile robot: the Moving Base

control method based on feedback linearization is presented which handles the overactuatedness by regarding the MB as four independent unicycles. Although the results of this controller were promising, they can be further improved by taking tire behavior into account in the control design. Tires introduce slip effects that compromise the position accuracy. A possible approach for counteracting this effect is to incorporate a tire model, well known in the field of automotive engineering [3], in a feedback linearization based controller as commonly used in the field of robotics [4], [5].

\section{MB CHARACTERISTICS}

Table I summarizes the main MB characteristics relevant to the control design. The high acceleration levels together with the considerable mass as mentioned in the table, lead to a significant weight transfer, influencing the actual vertical load of the tire. The friction force that a tire can deliver is in turn approximately proportional to the actual vertical load of the tire [3]. Consequently, the drive torque distribution across the four wheels should correspond to the actual vertical loads

TABLE I

MOVING BASE SPECIFICATIONS

\begin{tabular}{|l||l|}
\hline Vehicle mass with/without body & $756 / 694 \mathrm{~kg}$ \\
\hline Wheel base $\times$ track width & $1.4 \mathrm{~m} \times 1.4 \mathrm{~m}$ \\
\hline Center of gravity height & $0.40 \mathrm{~m}$ \\
\hline Maximum velocity & $50 \mathrm{~km} / \mathrm{h}$ \\
\hline Maximum translation acceleration & $10 \mathrm{~m} / \mathrm{s}^{2}$ \\
\hline Maximum centripetal acceleration & $12 \mathrm{~m} / \mathrm{s}^{2}$ \\
\hline Installed power & $52 \mathrm{~kW}$ \\
\hline Steering angle range & {$\left[-350^{\circ}, 350^{\circ}\right]$} \\
\hline
\end{tabular}




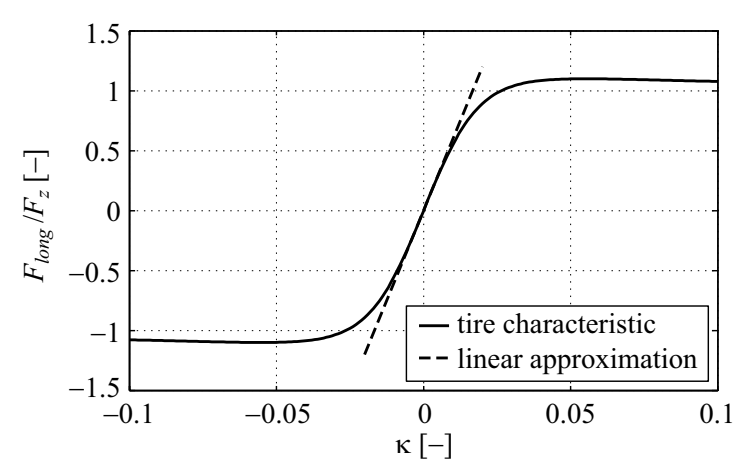

Fig. 2. The normalized longitudinal MB tire characteristic

in order to obtain the maximum performance of the $\mathrm{MB}$ in terms of acceleration and maneuverability.

The tire friction force also depends on the longitudinal slip $\kappa$ and the lateral slip angle $\alpha$, where $\kappa$ equals the normalized velocity difference between tire and road and $\alpha$ is the angle between the wheel plane and the velocity direction. This slip dependency is illustrated by Fig. 2 which shows the longitudinal tire force $F_{\text {long }}$, normalized by the vertical load $F_{z}$, as a function of the slip $\kappa$. The lateral force characteristic is described by a similar function. This characteristic justifies the incorporation of tire slip into the control design. Note that Fig. 2 also shows the linear approximation of the tire characteristic which is valid for $\kappa \ll 1$. The same type of approximation applies to the lateral characteristic.

\section{CONTROL CONCEPT}

The control objective of the MB is to let its center track a reference trajectory $\mathbf{q}_{r e f}$, consisting of the position in the $x, y$-plane and the orientation $\psi$ as a function of the time $t$ :

$$
\mathbf{q}_{\text {ref }}(t)=\left(\begin{array}{lll}
x_{\text {ref }}(t) & y_{\text {ref }}(t) \quad \psi_{\text {ref }}(t)
\end{array}\right)^{\mathrm{T}},
$$

where $\mathbf{q}_{\text {ref }}(t)$ must be continuously differentiable for a feasible trajectory. Note that (1) implies that the MB must be able to move in all directions.

The controller is designed using a similar approach as applied in [2], being inspired by the idea presented in [6], which is to decentralize the tracking problem. To this end, the reference vector $\mathbf{q}_{\text {ref }}$ is converted to reference positions $x_{\text {refij }}$ and $y_{\text {refij }}(i=f($ ront $), r($ ear $), j=l($ eft $), r(i g h t))$ for the wheels; refer to Fig. 3 depicting the MB coordinate systems. The reference positions for the wheels thus are:

$$
\begin{aligned}
x_{r e f f l} & =x_{r e f}+L_{d} \cos \left(\psi_{r e f}+\arctan (W / L)\right) \\
y_{r e f f l} & =y_{r e f}+L_{d} \sin \left(\psi_{r e f}+\arctan (W / L)\right) \\
x_{\text {reffr }} & =\cdots,
\end{aligned}
$$

where $L$ and $W$ are half the vehicle length and width respectively, and $L_{d}=\sqrt{L^{2}+W^{2}}$.

Although (2) uniquely defines the MB position and orientation, reference wheel orientation angles $\psi_{\text {refij }}$ are also needed because the MB must able to move in all directions, i.e. the MB must be fully controllable. These angles are

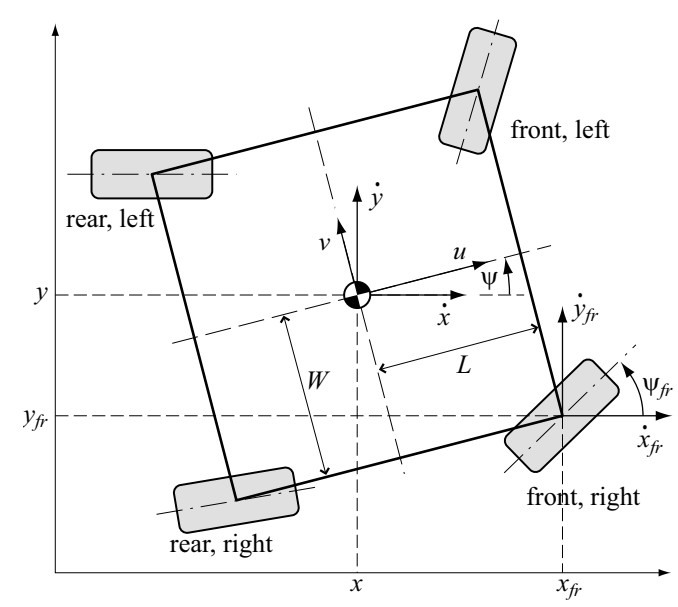

Fig. 3. The Moving Base coordinate systems

kinematically calculated according to:

$$
\psi_{\text {refij }}=\arctan \left(\frac{\dot{y}_{r e f i j}}{\dot{x}_{r e f i j}}\right) \text {, }
$$

where the velocities $\dot{x}_{\text {refij }}$ and $\dot{y}_{\text {refij }}$ are determined by differentiation of (2).

The MB is thus regarded as four independent systems, socalled unicycles, being single wheels that can be steered and driven. All four unicycles have their specific continuously differentiable reference signal $\mathbf{q}_{\text {refij }}(t)$ :

$$
\mathbf{q}_{\text {refij }}(t)=\left(\begin{array}{lll}
x_{\text {refij }}(t) & y_{\text {refij }}(t) & \psi_{\text {refij }}(t)
\end{array}\right)^{\mathrm{T}} .
$$

Consequently, four independent but identical tracking problems effectively remain.

As mentioned in section II, the mechanical coupling of the unicycles results in weight transfer when accelerating. This can be incorporated in the control design by implementing a varying unicycle mass $m_{i j}$, using Newton's second law:

$$
\begin{aligned}
& m_{f l}=\frac{m}{4}-\frac{h m}{4 L g}(\dot{u}-v \dot{\psi})-\frac{h m}{4 W g}(\dot{v}+u \dot{\psi}) \\
& m_{f r}=\frac{m}{4}-\frac{h m}{4 L g}(\dot{u}-v \dot{\psi})+\frac{h m}{4 W g}(\dot{v}+u \dot{\psi}) \\
& m_{r l}=\cdots,
\end{aligned}
$$

where $m$ is the total MB mass, $h$ is the center of gravity height, $g$ is the gravitational constant and $u$ and $v$ are the velocity components expressed in the local MB coordinate frame as depicted in Fig. 3. $(\dot{u}-v \dot{\psi})$ and $(\dot{v}+u \dot{\psi})$ are the longitudinal and lateral MB acceleration respectively.

It should be emphasized that the mechanical coupling of the unicycles is likely to cause disturbances. It is however assumed that these disturbances are small and rather well damped due to the tire compliance. This assumption is justified by the practical experiments (section VI).

The next step is to design a position controller for each unicycle. If tire slip is neglected, the robotics theory based on motion constraints [7] could be applied to formulate a unicycle model and subsequently design a feedback linearizing controller [4], [5]. This method, applied in [2], 
appears to yield rather acceptable results. One might however expect that taking tire slip into account will improve the characteristics of the controlled unicycles, and consequently the $\mathrm{MB}$, with respect to accuracy. Along this line of thinking, [8] and [9] provide a solution, based on the fact that tire dynamics are significantly faster than the WMR dynamics, leading to a so-called singular perturbation model. Using this model, the feedback linearization procedure is essentially straightforward although mathematically complicated. An explicit slip measurement appears not to be required, which is an advantage of the proposed controller. The resulting controller however has a rather complicated structure, providing limited insight. Moreover, the singular perturbation model incorporates the linearized tire characteristics, whereas extension to the nonlinear characteristics (Fig. 2) is far from straightforward. The next section therefore explores a different approach, based on a unicycle model taken from the field of automotive engineering and feedback linearization in a master-slave structure.

\section{UNiCyCle MODELING AND CONTROL}

Before developing a controller for the MB, this section first focuses on the modeling and control design for a unicycle.

\section{A. Modeling}

Based on the physical description of a tire as commonly used in the field of automotive engineering [3], this section will derive a unicycle model including a linear tire model with $1^{\text {st }}$-order dynamics. Note that longitudinal and lateral slip and the resulting forces are assumed to be independent, i.e. combined slip effects are ignored.

The equations of motion of unicycle $i j(i=f, r ; j=l, r)$ in the horizontal plane are:

$$
\begin{aligned}
& m_{i j}\left(\dot{u}_{i j}-v_{i j} \dot{\psi}_{i j}\right)=F_{l o n g i j} \\
& m_{i j}\left(\dot{v}_{i j}+u_{i j} \dot{\psi}_{i j}\right)=F_{l a t i j} \\
& I_{s} \ddot{\psi}_{i j}=T_{s i j},
\end{aligned}
$$

with longitudinal velocity $u_{i j}$, lateral velocity $v_{i j}$, heading angle $\psi_{i j}$, longitudinal force $F_{l o n g i j}$, and lateral force $F_{l a t i j}$. $I_{s}$ is the lumped inertia of the steering system and $T_{s i j}$ is the steer torque. Note that the subscripts $i$ and $j$ are omitted for those quantities that are identical for all four unicycles. Refer to Fig. 4 for a schematic illustration of the unicycle model (6) - (8).
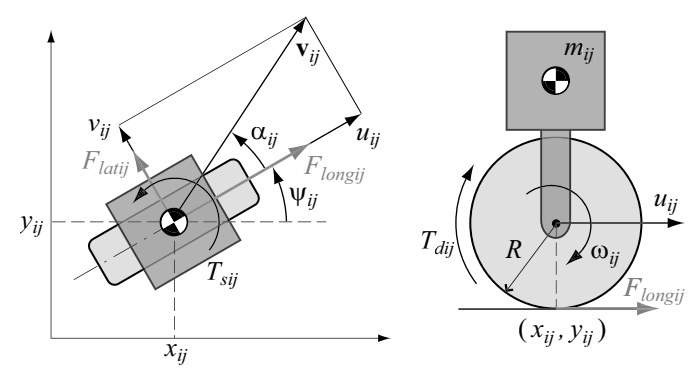

Fig. 4. The unicycle model, top view (left) and side view (right)
After linearization with respect to the longitudinal wheel slip $\kappa_{i j}$, the longitudinal force $F_{\text {longij }}$ can be expressed as:

$$
F_{\text {longij }}=K\left(F_{z i j}\right) \kappa_{i j}
$$

with longitudinal slip stiffness $K\left(F_{z i j}\right)$ where $F_{z i j}$ is the actual vertical force acting on wheel $i j . K\left(F_{z i j}\right)$ is approximately proportional to the vertical load $F_{z i j}=m_{i j} g$. Consequently, (9) can be rewritten into:

$$
F_{\text {longij }}=K_{n} m_{i j} \kappa_{i j}
$$

with normalized longitudinal slip stiffness $K_{n}$. Tire relaxation effects are represented by the following $1^{\text {st }}$-order differential equation for the longitudinal wheel slip:

$$
\sigma_{\kappa} \dot{\kappa}_{i j}+u_{i j} \kappa_{i j}=R \omega_{i j}-u_{i j}
$$

where $\sigma_{\kappa}$ is the longitudinal relaxation length, $R$ is the wheel radius and $\omega_{i j}$ the rotation velocity. Note that for steady state, (11) yields $\kappa_{i j}=\left(R \omega_{i j}-u_{i j}\right) / u_{i j}$, i.e. the normalized velocity difference between tire and road. The longitudinal tire model is completed by the dynamics due to the inertia $I_{d}$ of the tire/wheel/drive combination according to:

$$
I_{d} \dot{\omega}_{i j}=T_{d i j}-R F_{\text {longij }},
$$

where $T_{d i j}$ is the drive torque.

Similarly, the lateral tire force is approximated by a linear function of the slip angle $\alpha_{i j}$ :

$$
F_{l a t i j}=-C_{n} m_{i j} \alpha_{i j}
$$

with normalized lateral slip stiffness $C_{n}$. Introducing the lateral relaxation length $\sigma_{\alpha}, \alpha_{i j}$ is described by:

$$
\sigma_{\alpha} \dot{\alpha}_{i j}+u_{i j} \alpha_{i j}=v_{i j}
$$

where $\tan \alpha_{i j}$ is approximated by $\alpha_{i j}$, assuming small slip angles (not to be confused with possible large steering angles). For steady state, (14) yields $\alpha_{i j}=v_{i j} / u_{i j}$, which is indeed the tangent of the angle of the wheel velocity with respect to the wheel plane.

Summarizing, the complete unicycle model reads:

$$
\begin{aligned}
& \dot{x}_{i j}=u_{i j} \cos \psi_{i j}-v_{i j} \sin \psi_{i j} \\
& \dot{u}_{i j}=K_{n} \kappa_{i j}+v_{i j} \dot{\psi}_{i j} \\
& \dot{\kappa}_{i j}=\frac{R \omega_{i j}-u_{i j}-u_{i j} \kappa_{i j}}{\sigma_{\kappa}} \\
& \dot{\omega}_{i j}=\frac{T_{d i j}-R K_{n} m_{i j} \kappa_{i j}}{I_{d}} \\
& \dot{y}_{i j}=u_{i j} \sin \psi_{i j}+v_{i j} \cos \psi_{i j} \\
& \dot{v}_{i j}=-C_{n} \alpha_{i j}-u_{i j} \dot{\psi}_{i j} \\
& \dot{\alpha}_{i j}=\frac{v_{i j}-u_{i j} \alpha_{i j}}{\sigma_{\alpha}} \\
& \ddot{\psi}_{i j}=\frac{T_{s i j}}{I_{s}}
\end{aligned}
$$

where $\left(x_{i j}, y_{i j}\right)$ is the position of the center of gravity (Fig. 4). This model can be written in the following form:

$$
\dot{\mathbf{q}}_{i j}=\mathbf{f}\left(\mathbf{q}_{i j}\right)+\mathbf{g}\left(\mathbf{q}_{i j}\right) \mathbf{u}_{i j},
$$


with state vector $\mathbf{q}_{i j}=\left(x_{i j} u_{i j} \kappa_{i j} \omega_{i j} y_{i j} v_{i j} \alpha_{i j} \psi_{i j} \dot{\psi}_{i j}\right)^{\mathrm{T}}$ and input vector $\mathbf{u}_{i j}=\left(T_{d i j} T_{s i j}\right)^{\mathrm{T}}$. The vector functions $\mathbf{f}$ and $\mathrm{g}$ follow from (15) - (22).

\section{B. Control Design}

The unicycle controller will be based on input-output linearization by time-invariant state feedback [10], the advantage of this approach being that it (partly) linearizes the system and at the same time decouples a MIMO system. A necessary condition for input-output linearization is that the system must be square. Consequently, two outputs have to be defined. A possible choice for the unicycle output function $\mathbf{z}_{1 i j}$ is:

$$
\mathbf{z}_{1 i j}=\mathbf{h}\left(\mathbf{q}_{i j}\right)=\left(\begin{array}{c}
x_{i j}+l_{c p} \cos \psi_{i j} \\
y_{i j}+l_{c p} \sin \psi_{i j}
\end{array}\right),
$$

with $\mathbf{h}: \mathbb{R}^{n} \rightarrow \mathbb{R}^{l}$, where $n=9$ is the number of states, $l=2$ is the number of outputs and $l_{c p}>0$. This choice can be motivated from a physical point of view: instead of controlling the position and the heading of the center of gravity of the unicycle, the position of a virtual control point $V_{c p i j}$ is controlled. This control point is located at distance $l_{c p}$ in front of the center of gravity, see Fig. 5, which guarantees that not only the position $\left(x_{i j}, y_{i j}\right)$, but also the heading $\psi_{i j}$ converge to their reference values as long as the forward velocity $u_{i j}$ is nonzero and the controlled system is stable. $l_{c p}$ is in fact a tuning parameter, primarily influencing the damping of the controlled system.

Input-output linearization is basically performed by differentiating the outputs with respect to time until both inputs 'appear' and then inverting the input-output relation. The number of differentiations of output $z_{1 i j k}(k=1,2)$ necessary for at least one input to appear, is called the relative degree $r_{k}$. For the unicycle model, $r_{1}$ and $r_{2}$ are both equal to 2 . It appears however that only the second input $T_{s i j}$ is then visible in both outputs, which renders the system non-linearizable by state feedback ${ }^{1}$. The solution adopted here is to reduce the model by taking $\kappa_{i j}$ instead of $T_{d i j}$ as input, thereby removing (17) and (18) from the

\footnotetext{
${ }^{1}$ Only after four differentiations, the input $T_{d i j}$ appears in the outputs. Consequently, the $2^{\text {nd }}$ time derivative $\ddot{T}_{s i j}$ then also appears in the outputs. Defining $\ddot{T}_{s i j}$ as a new input would provide a solution, known as dynamic extension. This would however increase the system order, complicating the feedback control design.
}

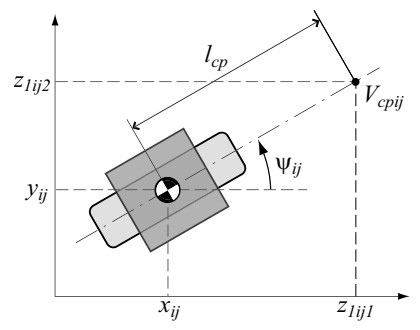

Fig. 5. The virtual control point $V_{c p i j}$ model. Consequently the number of states reduces to $n=7$ whereas the inputs are redefined:

$$
\begin{aligned}
\mathbf{q}_{i j} & =\left(\begin{array}{lllllll}
x_{i j} & u_{i j} & y_{i j} & v_{i j} & \alpha_{i j} & \psi_{i j} & \dot{\psi}_{i j}
\end{array}\right)^{\mathrm{T}} \\
\mathbf{u}_{i j} & =\left(\begin{array}{lll}
\kappa_{i j} & T_{s i j}
\end{array}\right)^{\mathrm{T}} .
\end{aligned}
$$

Because the real input of the unicycle remains $T_{d i j}$, a slave controller is needed which controls $\kappa_{i j}$ using $T_{d i j}$. In the remainder of this section, first the position controller for the reduced unicycle model is designed, after which the $\kappa$ controller will shortly be described.

Since $r_{k}=2(k=1,2)$, the output vector $\mathbf{z}_{1 i j}$ has to be differentiated twice in order to arrive at the linearized model. With the Lie derivative $L_{f} h_{k}\left(\mathbf{q}_{i j}\right)$ defined by:

$$
L_{f} h_{k}\left(\mathbf{q}_{i j}\right) \triangleq \frac{\partial h_{k}\left(\mathbf{q}_{i j}\right)}{\partial \mathbf{q}_{i j}} \mathbf{f}\left(\mathbf{q}_{i j}\right),
$$

the first derivative $\dot{\mathbf{z}}_{1 i j}$ can be formulated as:

$$
\begin{aligned}
\dot{\mathbf{z}}_{1 i j} & =\left(\begin{array}{c}
L_{f} h_{1}\left(\mathbf{q}_{i j}\right) \\
L_{f} h_{2}\left(\mathbf{q}_{i j}\right)
\end{array}\right) \\
& =\left(\begin{array}{c}
u_{i j} \cos \psi_{i j}-\left(v_{i j}+l_{c p} \dot{\psi}_{i j}\right) \sin \psi_{i j} \\
u_{i j} \sin \psi_{i j}+\left(v_{i j}+l_{c p} \dot{\psi}_{i j}\right) \cos \psi_{i j}
\end{array}\right) .
\end{aligned}
$$

Introducing a state vector $\mathbf{z}_{2 i j}=\dot{\mathbf{z}}_{1 i j}$, the second derivative equals:

$$
\dot{\mathbf{z}}_{2 i j}=\mathbf{b}\left(\mathbf{q}_{i j}\right)+\mathbf{H}\left(\mathbf{q}_{i j}\right) \mathbf{u}_{i j}
$$

with

$$
\begin{aligned}
\mathbf{b}\left(\mathbf{q}_{i j}\right) & =\left(\begin{array}{c}
L_{f}^{2} h_{1}\left(\mathbf{q}_{i j}\right) \\
L_{f}^{2} h_{2}\left(\mathbf{q}_{i j}\right)
\end{array}\right) \\
& =\left(\begin{array}{c}
C_{n} \alpha_{i j} \sin \psi_{i j}-l_{c p} \dot{\psi}_{i j}^{2} \cos \psi_{i j} \\
-C_{n} \alpha_{i j} \cos \psi_{i j}-l_{c p} \psi_{i j}^{2} \sin \psi_{i j}
\end{array}\right),
\end{aligned}
$$

and

$$
\begin{aligned}
\mathbf{H}\left(\mathbf{q}_{i j}\right) & =\left(\begin{array}{cc}
L_{g_{1}} L_{f} h_{1}\left(\mathbf{q}_{i j}\right) & L_{g_{2}} L_{f} h_{1}\left(\mathbf{q}_{i j}\right) \\
L_{g_{1}} L_{f} h_{2}\left(\mathbf{q}_{i j}\right) & L_{g_{2}} L_{f} h_{2}\left(\mathbf{q}_{i j}\right)
\end{array}\right) \\
& =\left(\begin{array}{cc}
K_{n} \cos \psi_{i j} & -\frac{l_{c p}}{I_{s}} \sin \psi_{i j} \\
K_{n} \sin \psi_{i j} & \frac{l_{c p}}{I_{s}} \cos \psi_{i j}
\end{array}\right) .
\end{aligned}
$$

This clearly shows that the inputs appear in the differential equation after two differentiations. Note that the determinant $|\mathbf{H}|=K_{n} l_{c p} / I_{s}$ must be nonzero because the inverse $\mathbf{H}^{-1}$ will be applied in the design. Consequently, $l_{c p}$ must be nonzero which can be readily understood because the wheel orientation would be undefined if $V_{c p i j}$ is located in the wheel center.

The differential equations (28), (29) in fact provide a new description of the linearizable part of the reduced unicycle model. The order of this subsystem equals $\sum_{k=1}^{l} r_{k}=4$. Since the order of the reduced model equals 7, a subsystem of order 3 remains that cannot be linearized. Denoting the state of this subsystem by $\mathbf{z}_{3 i j}$, a possible choice for this state is $\mathbf{z}_{3 i j}=\left(\begin{array}{lll}v_{i j} & \alpha_{i j} & \dot{\psi}_{i j}\end{array}\right)^{\mathrm{T}}$ which after differentiation results in an expression of the form:

$$
\dot{\mathbf{z}}_{3 i j}=\mathbf{r}\left(\mathbf{z}_{1 i j}, \mathbf{z}_{2 i j}, \mathbf{z}_{3 i j}, \mathbf{u}_{i j}\right),
$$


with $\mathbf{r}: \mathbb{R}^{7} \rightarrow \mathbb{R}^{3}$. With (28), (29) and (32), the reduced unicycle model is now rewritten in the so-called normal form.

The actual feedback linearization is obtained by choosing the input $\mathbf{u}_{i j}$ according to the following feedback law:

$$
\mathbf{u}_{i j}=\mathbf{H}^{-1}\left(\mathbf{q}_{i j}\right)\left(\boldsymbol{v}_{i j}-\mathbf{b}\left(\mathbf{q}_{i j}\right)\right)
$$

which finally results in the unicycle model:

$$
\begin{aligned}
\dot{\mathbf{z}}_{1 i j} & =\mathbf{z}_{2 i j} \\
\dot{\mathbf{z}}_{2 i j} & =\mathbf{v}_{i j} \\
\dot{\mathbf{z}}_{3 i j} & =\mathbf{r}\left(\mathbf{z}_{1 i j}, \mathbf{z}_{2 i j}, \mathbf{z}_{3 i j}, \mathbf{v}_{i j}\right),
\end{aligned}
$$

with new external input $\boldsymbol{v}_{i j}$. The model (34) - (36) shows that the dynamics of the reduced unicycle model have now been decomposed into a linear decoupled input-output part with states $\mathbf{z}_{1 i j}$ and $\mathbf{z}_{2 i j}$ and a nonlinear "unobservable" part with state $\mathbf{z}_{3 i j}$, generally referred to as the internal dynamics.

Tracking behavior of the linear input-output dynamics is obtained by a regular PD-controller with feedforward:

$$
\begin{aligned}
\boldsymbol{v}_{i j}=\ddot{\mathbf{z}}_{1 r e f i j}+\mathbf{K}_{d}\left(\dot{\mathbf{z}}_{1 r e f i j}-\dot{\mathbf{z}}_{1 i j}\right) \\
+\mathbf{K}_{p}\left(\mathbf{z}_{1 \text { refij }}-\mathbf{z}_{1 i j}\right),
\end{aligned}
$$

where $\mathbf{K}_{p}$ and $\mathbf{K}_{d}$ are diagonal $2 \times 2$ matrices containing the proportional and differential gains respectively. In order to obtain equal dynamic behavior in longitudinal and lateral direction, both proportional gains are equal, as are both differential gains. $\mathbf{z}_{1 \text { refij }}$ is calculated by substituting the reference trajectories (2) and (3) into (24). The resulting expression can subsequently be differentiated in order to obtain $\dot{\mathbf{z}}_{1 \text { refij }}$ and $\ddot{\mathbf{z}}_{1 \text { refij }}$.

The controller (37) stabilizes the input-output dynamics. In order to prevent from undesirable phenomena, the internal dynamics however must also be stable or, in other words, the system should be minimum phase in the nonlinear sense. An example of such a phenomenon is lateral oscillation of the unicycle wheel while the control point $V_{c p i j}$ 'perfectly' tracks the reference trajectory. Due to the nonlinearity however, the stability has to be checked for each reference trajectory. In case the unicycle is driving along a straight line with a constant forward velocity, the asymptotic stability of the internal dynamics can be proven for $u_{i j}>0$ and $l_{c p}>\sigma_{\alpha}=0.22 \mathrm{~m}$. The requirement $u_{i j}>0$ can be understood from a physical point of view: when the unicycle is standing still, the heading angle $\psi_{i j}$ does not converge to the reference value.

Finally, the slip controller is designed. The dynamics between the drive torque $T_{d i j}$ and the longitudinal slip $\kappa_{i j}$ are described by (17) and (18), where the forward velocity $u_{i j}$ is regarded as a relatively slowly varying parameter. Note that the longitudinal tire dynamics are thus assumed to be significantly faster than the WMR dynamics, which is the same fundamental idea as used in [9]. As (17), (18) is a linear SISO system, a regular PD controller can be used:

$$
T_{d i j}=K_{d \kappa}\left(\dot{\kappa}_{r e f i j}-\dot{\kappa}_{i j}\right)+K_{p \kappa}\left(\kappa_{r e f i j}-\kappa_{i j}\right),
$$

with differential gain $K_{d \kappa}$ and proportional gain $K_{p \kappa} . \kappa_{r e f i j}$ is the longitudinal slip reference generated by (33).

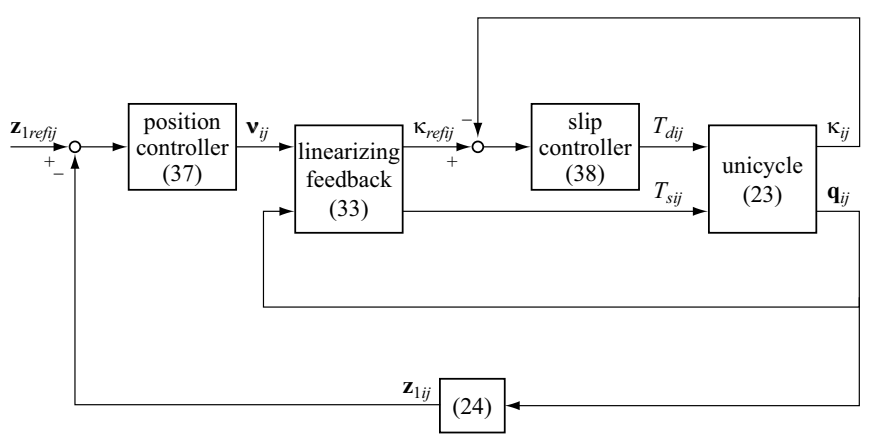

Fig. 6. Block scheme of the controlled unicycle

Summarizing, the controller developed in this section consists of a slave slip controller, a linearizing feedback loop and a master position controller. The resulting block scheme is depicted in Fig. 6.

\section{Multicycle Control Design}

Having designed a unicycle controller, the MB controller can now be established. As explained in section III, the MB controller consists of multiple identical unicycle controllers. It is therefore called the multicycle controller. One small adaptation however must be made because the wheels have to be steered relative to the MB body only.

The unicycle steering torque $T_{s i j}$ equals $I_{s} \ddot{\psi}_{i j}$ for an ideal (frictionless) unicycle. Because however the MB-body also rotates around its vertical axis with angular acceleration $\ddot{\psi}$, the net required steering torque $\tilde{T}_{s i j}$ for the multicycle is:

$$
\begin{aligned}
\tilde{T}_{s i j} & =I_{s} \ddot{\delta}_{i j}=I_{s}\left(\ddot{\psi}_{i j}-\ddot{\psi}\right) \\
& =T_{s i j}-I_{s} \ddot{\psi}
\end{aligned}
$$

where $\delta_{i j}$ is the steering angle, i.e. the wheel orientation with respect to the MB body. As a consequence, a compensation term $-I_{s} \ddot{\psi}$ has to be added to $T_{s i j}$ to obtain the multicycle steering torque. The MB controller is now fully determined.

\section{FIRST EXPERIMENTS}

After having tuned and evaluated the designed controller on a simulation level, the final step in the control design entails testing the controller in reality. To this end, an 'eightshaped' test trajectory is applied, shown in Fig. 7. The centripetal acceleration during cornering is $9 \mathrm{~m} / \mathrm{s}^{2}$, illustrating the MB behavior with a constant lateral tire slip. The velocity

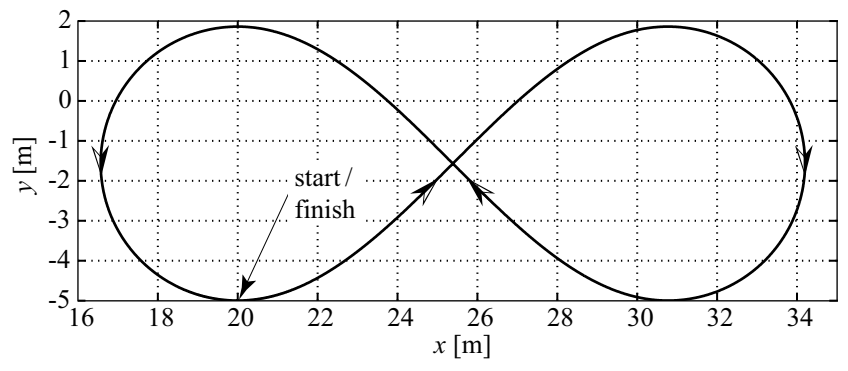

Fig. 7. The reference trajectory in the $x, y$-plane 
tangential to the track is increased from 0 to $20 \mathrm{~km} / \mathrm{h}$ with an acceleration of $5 \mathrm{~m} / \mathrm{s}^{2}$, then kept constant and finally decreased again to zero with $-5 \mathrm{~m} / \mathrm{s}^{2}$ acceleration. The desired MB orientation is directed tangentially to the track.

The test trajectory is applied to a simulation model of the controlled MB as well as to the real MB. The simulation model comprises a comprehensive physical model of the MB, implemented together with the controller in MATLAB/Simulink ${ }^{\mathrm{TM}}$. The feedback linearizing controller requires all MB states to be estimated or measured. To this end, a discrete Kalman filter is used to estimate position and velocity in two directions, using an on-board acceleration sensor, wheel velocity and steering angle encoders as well as a regularly spaced grid of magnets in the road surface. The lateral and longitudinal slip - the latter being used in the slip controller - are estimated using two dedicated nonlinear observers. All observers are implemented in the simulation model as well as in the real-time MB control system.

Fig. 8 shows the first results of both the simulation and the practical experiment, displaying the $x$-position error $e_{x}=x_{r e f}-x$, the $y$-position error $e_{y}=y_{r e f}-y$ and the orientation error $e_{\psi}=\psi_{\text {ref }}-\psi$ for the MB center as a function of time. These errors are calculated based on the positions estimated by the observer (in the model as well as in reality) whereas the yaw angle is a direct measurement.

The noticeable differences between the simulated and the measured errors are due to model uncertainties and simplifications, especially with respect to the tire characteristics and the floor flatness. It should however be noted that Fig. 8 shows the error signals. Nevertheless, the simulation and the experiment show corresponding tendencies.

It can be concluded that the position error in the practical experiment is reasonable $\left(\left|e_{x}\right|<0.3 \mathrm{~m},\left|e_{y}\right|<0.3 \mathrm{~m}\right.$ and $\left|e_{\psi}\right| \leq 0.1 \mathrm{rad}$ ), given the high centripetal acceleration during the cornering part of the trajectory. The controller gains in (37) and (38) are however not yet optimized with respect
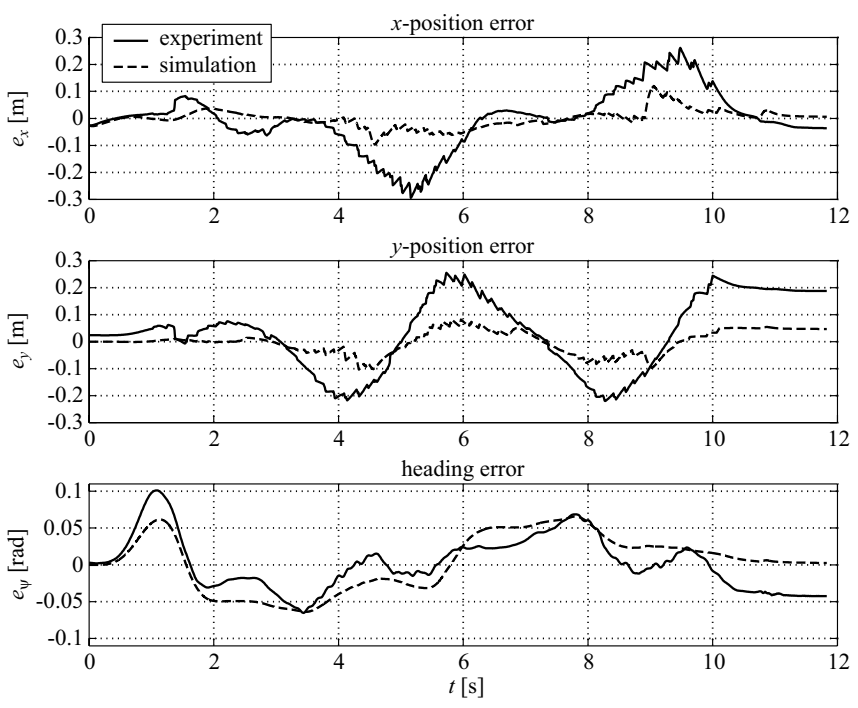

Fig. 8. Simulation and experiment results to these errors; further improvement might therefore be expected. Finally, it is noted that the steady state errors in the simulation as well as in reality are caused by the fact that the internal dynamics are stable but not asymptotically stable for $u_{i j}=0$, allowing for a final non-zero value of each wheel orientation $\psi_{i j}$. Because however $\mathbf{z}_{1 i j}$ does converge to $\mathbf{z}_{1 \text { refij }}$, these orientation errors must be 'compensated for' by corresponding errors of the MB center.

\section{CONCLUSIONS}

Summarizing, the main conclusion is that the multicycle controller incorporating a linearized tire slip characteristic successfully controls the wheeled mobile robot, even in high dynamic trajectories. In the multicycle approach, the overactuatedness of the robot is employed to optimize the drive torque distribution across the wheels. The resulting controller can easily be adapted to other platform configurations. It is however necessary to have the longitudinal and lateral slip available, which requires a slip observer. The current approach allows for a relatively straightforward extension to the nonlinear tire characteristic.

Besides further tuning of the controller gains, a possible improvement of the multicycle approach lies in the fact that tire slip is still neglected on the multicycle level, namely at the kinematic determination of the reference steering angles. Furthermore, the nonlinear tire characteristic needs to be incorporated in order to achieve a more accurate behavior at high longitudinal and lateral accelerations. Finally, a thorough evaluation of the designed controller - compared to the one without tire slip designed earlier - is desired in order to determine the level of improvement. These issues will be the subject of further research.

\section{REFERENCES}

[1] O. J. Gietelink, J. Ploeg, B. de Schutter, and M. Verhaegen, "Development of advanced driver assistance systems with vehicle hardwarein-the-loop simulations," Vehicle System Dynamics, vol. 44, no. 7, pp. 569-590, July 2006.

[2] J. Ploeg, J. P. M. Vissers, and H. Nijmeijer, "Control design for an overactuated wheeled mobile robot," in Proceedings of the 4th IFAC Symposium on Mechatronic Systems, Heidelberg, Germany, Sept. 1214, 2006.

[3] H. B. Pacejka, Tire and Vehicle Dynamics. Oxford: ButterworthHeinemann, 2002.

[4] C. Canudas de Wit, B. Siciliano, and G. Bastin, Theory of Robot Control. London: Springer-Verlag, 1996.

[5] J. D. Bendtsen, P. Anderson, and T. S. Pedersen, "Robust feedbacklinearization based control design for a wheeled mobile robot," in Proceedings of the 6th International Symposium on Advanced Vehicle Control, Hiroshima, Japan, Sept. 9-13, 2002.

[6] J. Borenstein, "Control and kinematic design of multi-degree-offreedom mobile robots with compliant linkage," IEEE Trans. Robot. Automat., vol. 11, no. 1, pp. 21-35, 1995.

[7] G. Campion, G. Bastin, and B. D' Andrea-Novel, "Structural properties and classification of kinematic and dynamic models of wheeled mobile robots," IEEE Trans. Robot. Automat., vol. 12, no. 1, pp. 47-62, 1996.

[8] B. D'Andréa-Novel, G. Campion, and G. Bastin, "Control of wheeled mobile robots not satisfying ideal velocity constraints: a singular perturbation approach," International Journal of Robust and Nonlinear Control, vol. 5, pp. 243-267, 1995.

[9] I. Motte and G. Campion, "A slow manifold approach for the control of mobile robots not satisfying the kinematic constraints," IEEE Trans. Robot. Automat., vol. 16, no. 6, pp. 875-880, Dec. 2000.

[10] H. Nijmeijer and A. J. van der Schaft, Nonlinear Dynamical Control Systems. New York: Springer-Verlag, 1990. 